\title{
On a sum involving the number of distinct prime factors function related to the integer part function
}

\author{
Mihoub Bouderbala ${ }^{1}$ and Meselem Karras ${ }^{2}$ \\ ${ }^{1}$ Institute of Mathematics-USTHB, LA3C \\ Houari-Boumédiène University of Science and Technology \\ Bab Ezzouar, Algeria \\ e-mail: mi houb 75bouder@gmail .com \\ ${ }^{2}$ Djilali Bounaama Khemis Miliana University \\ FIMA Laboratory, Algeria \\ e-mail: karras.mehotmail.fr
}

Received: 15 January $2020 \quad$ Revised: 18 November $2020 \quad$ Accepted: 19 November 2020

\begin{abstract}
In this paper, we obtain asymptotic formula on the sum $\sum_{n \leq x} \omega\left(\left\lfloor\frac{x}{n}\right\rfloor\right)$, where $\omega(n)$ denote the number of distinct prime divisors of $n$ and $\lfloor t\rfloor$ denotes the integer part of $t$.
\end{abstract}

Keywords: Number of distinct prime divisors, Mean value, Integer part.

2010 Mathematics Subject Classification: 11N37, 11A25, 11N36.

\section{Introduction}

Let, as usual, for an integer $n \geq 1, \omega(n):=\sum_{p \mid n} 1$ denote the the number of distinct prime divisors of $n$. Many authors investigated the properties of this function. In 1917, G. H. Hardy and S. Ramanujan [4] proved the classical result,

$$
\sum_{n \leq x} \omega(n)=x \log \log x+B x+O\left(\frac{x}{\log x}\right),
$$

such that $B=\gamma+\sum_{p}(\log (1-1 / p)+1 / p)$ and $\gamma$ is Euler's constant. The result (1) was generalized in 1970 [6] and in 1976 [3] by the following formula 


$$
\sum_{k \leq n} \omega(k)=n \log \log n+B n+\sum_{j=1}^{m} \frac{n a_{j}}{(\log n)^{j}}+O\left(\frac{n}{(\log n)^{m+1}}\right),
$$

for all integer $m \geq 1$, with

$$
a_{j}=-\int_{1}^{\infty} \frac{\{t\}}{t^{2}}(\log t)^{j-1} d t
$$

In [5], we find another interesting result

$$
\sum_{n \leq x} \omega(d(n))=c x+O\left(x^{1 / 2} \log ^{5} x\right)
$$

such that $d(n)$ is the number of divisors of $n$ and $c>0$ it's a constant. It is easy to show that the following relationship is correct for all real $x \geq 1$

$$
\sum_{n \leq x} d(n)=\sum_{n \leq x}\left\lfloor\frac{x}{n}\right\rfloor
$$

where $\lfloor t\rfloor$ denotes the integer part of any $t \in \mathbb{R}$ ( see [2, example 4.18] ).

The possible question is what are the similarities between the mean values of the functions $\omega(d(n))$ and $\omega\left(\left\lfloor\frac{x}{n}\right\rfloor\right)$ ? Since, the sum is on a less dense set than the first, it is obvious that the result will be at least with an error term lower, than what is given in the formula (3).

\section{Main result}

In this section, we establish a result concerning the mean value of the function $\omega\left(\left\lfloor\frac{x}{n}\right\rfloor\right)$. More precisely, we prove the following theorem:

Theorem 1. For all $x \geq 1$ large enough, we have

$$
\sum_{n \leq x} \omega\left(\left\lfloor\frac{x}{n}\right\rfloor\right)=C x+O\left(x^{1 / 2} \log x\right) .
$$

Such that $C \approx 0.5918 \cdots$.

The proof of this result is based on the following lemmas:

Lemma 1. Let $x \geq 1$ be real number. For any arithmetic function $f$ we have

$$
\sum_{n \leq x} f\left(\left\lfloor\frac{x}{n}\right\rfloor\right)=\sum_{n \leq x} f(n)\left(\left\lfloor\frac{x}{n}\right\rfloor-\left\lfloor\frac{x}{n+1}\right\rfloor\right) .
$$

Proof. If we pose $\left\lfloor\frac{x}{n}\right\rfloor=k$, then we have the following equivalents:

$$
\left\lfloor\frac{x}{n}\right\rfloor=k \Longleftrightarrow x / n-1<k \leq x / n \Longleftrightarrow x /(k+1)<n \leq x / k .
$$


Using that, we get

$$
\begin{aligned}
\sum_{n \leq x} f\left(\left\lfloor\frac{x}{n}\right\rfloor\right) & =\sum_{\substack{n \leq x \\
x / n-1<k \leq x / n}} f(k) \\
& =\sum_{\substack{k \leq x \\
x /(k+1)<n \leq x / k}} f(k) \\
& =\sum_{\substack{k \leq x \\
n \leq x / k}} f(k)-\sum_{\substack{k \leq x \\
n \leq x /(k+1)}} f(k) \\
& =\sum_{k \leq x} \sum_{n \leq x / k} f(k)-\sum_{k \leq x} \sum_{n \leq x /(k+1)} f(k) \\
& =\sum_{k \leq x} f(k) \sum_{n \leq x / k} 1-\sum_{k \leq x} f(k) \sum_{n \leq x /(k+1)} 1 \\
& =\sum_{k \leq x} f(k)\left(\left\lfloor\frac{x}{k}\right\rfloor-\left\lfloor\frac{x}{k+1}\right\rfloor\right) .
\end{aligned}
$$

Lemma 2. Let $n \in \mathbb{Z}_{\geq 0}$ and $\delta>0$ real. For all real $x \geq 1$, we have

$$
\int_{x}^{+\infty} e^{-\delta t}(\log t)^{n} d t \leq \frac{n !}{\delta} e^{-\delta x}\left(\log x+\frac{1}{\delta x}\right)^{n} .
$$

Proof. We put $I_{n}=\int_{x}^{+\infty} e^{-\delta t}(\log t)^{n} d t$ and we use integration by parts, so

$$
I_{n}=\frac{e^{-\delta x}}{\delta}(\log x)^{n}+\frac{n}{\delta} \int_{x}^{+\infty} \frac{(\log t)^{n-1}}{t e^{\delta t}} d t \leq \frac{e^{-\delta x}}{\delta}(\log x)^{n}+\frac{n}{\delta x} I_{n-1} .
$$

And by recurrence, we get

$$
\begin{aligned}
I_{n} & \leq \frac{e^{-\delta x}}{\delta} \sum_{k=0}^{n} k !\left(\begin{array}{c}
n \\
k
\end{array}\right) \frac{(\log x)^{n-k}}{(\delta x)^{k}} \\
& \leq \frac{n !}{\delta} e^{-\delta x}\left(\log x+\frac{1}{\delta x}\right)^{n} .
\end{aligned}
$$

Lemma 3. Let $x$ be sufficiently large, there is a constant $C>0$ such that

$$
\sum_{n \leq x} \frac{\omega(n)}{n(n+1)}=C+O\left(\frac{\log \log x}{x}\right),
$$

such that $C \approx 0.5918 \ldots$

Proof. Let $x \geq 2$, we have

$$
\sum_{n \leq x} \frac{\omega(n)}{n(n+1)}=\sum_{n \geq 1} \frac{\omega(n)}{n(n+1)}-\sum_{n>x} \frac{\omega(n)}{n(n+1)} .
$$

Now the well-known trivial bound of $\omega(n)$, applied to the first sum on the right-hand side of (5), implies that 


$$
\sum_{n \geq 1} \frac{\omega(n)}{n(n+1)} \leq \frac{1}{\log 2} \sum_{n \geq 1} \frac{\log n}{n(n+1)}
$$
find

We deduce that the series $\sum_{n \geq 1} \frac{\omega(n)}{n(n+1)}$ is convergent, and with a numerical calculation, we

$$
C=\sum_{n \geq 1} \frac{\omega(n)}{n(n+1)} \approx 0.5918 \ldots
$$

In the last sum in $(5)$, we put $g(t)=\frac{1}{t(t+1)}$, and by partial summation, we have

$$
\begin{aligned}
\sum_{n>x} \frac{\omega(n)}{n(n+1)} & =-g(x) \sum_{n \leq x} \omega(n)-\int_{x}^{+\infty} g^{\prime}(t)\left(\sum_{x<n \leq t} \omega(n)\right) d t \\
& =\frac{-1}{x(x+1)} \sum_{n \leq x} \omega(n)+\int_{x}^{+\infty} \frac{2 t-1}{t^{2}(t+1)^{2}}\left(\sum_{x<n \leq t} \omega(n)\right) d t .
\end{aligned}
$$

And from (1) we obtain,

$$
\left|\sum_{n>x} \frac{\omega(n)}{n(n+1)}\right| \leq \frac{\log \log x}{x}+\frac{B}{x}+O\left(\frac{1}{x \log x}\right)+O\left(\int_{x}^{+\infty} \frac{\log \log t}{t^{2}} d t\right) .
$$

So, by Lemma $2(n=1, \delta=1)$, and using a variable change, we find

$$
\begin{aligned}
\int_{x}^{+\infty} \frac{\log \log t}{t^{2}} d t & \leq \frac{\log \log x}{x}+\frac{1}{x \log x} \\
& =O\left(\frac{\log \log x}{x}\right)
\end{aligned}
$$

Finally, using the last estimate in (7), we get

$$
\sum_{n>x} \frac{\omega(n)}{n(n+1)}=O\left(\frac{\log \log x}{x}\right),
$$

and collecting (8), (6) and (5), we get the following desired result.

Lemma 4. For all $x \geq 1$, we have

$$
\sum_{n \geq 0}\left|\left\{\frac{x}{n+1}\right\}-\left\{\frac{x}{n+2}\right\}\right|=\frac{2}{\pi} \zeta(3 / 2) x^{1 / 2}+O\left(x^{2 / 5}\right)
$$

where $\{t\}$ denotes the fractional part of any $t \in \mathbb{R}$.

Proof. The proof of this result is found in the paper [1].

Proof of the theorem. For all $x \geq 1$, by Lemma 1, we have

$$
\begin{aligned}
\sum_{n \leq x} \omega\left(\left\lfloor\frac{x}{n}\right\rfloor\right) & =\sum_{n \leq x} \omega(n)\left(\left\lfloor\frac{x}{n}\right\rfloor-\left\lfloor\frac{x}{n+1}\right\rfloor\right) \\
& =x \sum_{n \leq x} \frac{\omega(n)}{n(n+1)}+\sum_{n \leq x} \omega(n)\left(\left\{\frac{x}{n+1}\right\}-\left\{\frac{x}{n}\right\}\right),
\end{aligned}
$$


on the other hand, by trivial bound of $\omega(n)$ and Lemma 4, we have

$$
\begin{aligned}
\left|\sum_{n \leq x} \omega(n)\left(\left\{\frac{x}{n}\right\}-\left\{\frac{x}{n+1}\right\}\right)\right| & \leq \sum_{n \leq x} \omega(n)\left|\left\{\frac{x}{n}\right\}-\left\{\frac{x}{n+1}\right\}\right| \\
& \leq \frac{\log x}{\log 2} \sum_{n \geq 0}\left|\left\{\frac{x}{n+1}\right\}-\left\{\frac{x}{n+2}\right\}\right| \\
& =\frac{\log x}{\log 2}\left(\frac{2}{\pi} \zeta(3 / 2) x^{1 / 2}+O\left(x^{2 / 5}\right)\right) \\
& =\frac{2 \zeta(3 / 2)}{\pi \log 2} x^{1 / 2} \log x+O\left(x^{2 / 5} \log x\right) .
\end{aligned}
$$

Therefore,

$$
\sum_{n \leq x} \omega(n)\left(\left\{\frac{x}{n}\right\}-\left\{\frac{x}{n+1}\right\}\right)=O\left(x^{1 / 2} \log x\right) .
$$

Finally, by Lemma 3, we obtain

$$
\begin{aligned}
\sum_{n \leq x} \omega\left(\left\lfloor\frac{x}{n}\right\rfloor\right) & =C x+O(\log \log x)+O\left(x^{1 / 2} \log x\right) \\
& =C x+O\left(x^{1 / 2} \log x\right) .
\end{aligned}
$$

\section{Acknowledgements}

The authors thank Professor Olivier Bordellès for his help and attention to this work.

\section{References}

[1] Balazard, M. (2017). Sur la variation totale de la suite des parties fractionnaires des quotients d'un nombre réel positif par les nombres entiers naturels consécutifs, Mosc. J. Comb. Number Theory, 7, 3-23.

[2] Bordellès, O. (2012). Arithmetic Tales, Springer.

[3] Diaconis, (1976). Asymptotic expansions for the mean and variance of the number of prime factors of a number $n$, Technical Report No. 96, Department of Statistics, Stanford University.

[4] Hardy, G. H., \& Ramanujan, S. (1917). The normal number of prime factors of a number $n$. Quart. J. Math., 48, 76 - 92.

[5] Rieger, G. J. (1972). Uber einige arithmetische Summen. Manuscripta Math., 7, 23-34.

[6] Safari, B. (1970). Sur quelques applications de la méthode de l'hyperbole de Dirichlet a la théorie des nombres premiers, Enseignement Math., 14, 205-224. 